\title{
COMPORTAMIENTO PRODUCTIVO DE CONEJOS DURANTE EL PERÍODO DE CRECIMIENTO-ENGORDE ALIMENTADOS CON FRUTOS DE MANGO (MANGIFERA INDICA) EN SUSTITUCIÓN PARCIAL DEL ALIMENTO BALANCEADO COMERCIAL
}

\author{
PRODUCTIVE BEHAVIOR IN RABBITS DURING THE FATTENING GROWTH \\ PERIOD-FED WITH MANGO AS PARTIAL SUBSTITUTION OF THE \\ COMMERCIAL BALANCED FOOD
}

O. R. Palma ${ }^{1}$; E. A. Hurtado 2

\section{RESUMEN}

Con el fin de evaluar el comportamiento productivo de conejos durante el período de crecimiento-engorde, se realizó un experimento que consistió en la sustitución parcial del alimento balanceado comercial $(\mathrm{ABC})$ por el fruto de mango criollo (Mangifera indica) en una granja comercial del estado Monagas, Venezuela. El experimento tuvo una duración de 63 días, se utilizaron 32 conejos mestizos Nueva Zelanda x California de 30 días de edad alojados en jaulas. Se realizó un diseño completamente aleatorizado con cuatro tratamientos: $\mathrm{T} 1: 120 \mathrm{~g}$ de $\mathrm{ABC} ; \mathrm{T} 2: 80 \mathrm{~g}$ de $\mathrm{ABC}+40 \mathrm{~g}$ de mango criollo; $\mathrm{T} 3: 60 \mathrm{~g}$ de $\mathrm{ABC}+60 \mathrm{~g}$ de mango criollo y T4: $40 \mathrm{~g}$ de $\mathrm{ABC}+80 \mathrm{~g}$ de mango criollo. Las variables dependientes fueron: ganancia diaria de peso promedio (GDP), peso final a los 93 días de edad (PESOFIN), consumo total (CONSTOTAL) y conversión alimenticia con alimento balanceado comercial (CAABC). Se realizó un análisis de varianza por el método de mínimos cuadrados. Los resultados arrojaron diferencias significativas para las variables bajo estudio, siendo los mayores promedios en GDP para T1: 26,69 $\pm 1,34 \mathrm{~g}$; PESOFIN para T1: 2083,75 \pm 26,09 g; CONSTOTAL fue para T1: 7434, $37 \pm 6,80 \mathrm{~g}$. Las mejores CAABC se obtuvieron en los tratamientos que incorporaron el mango criollo. En general los resultados obtenidos permiten inferir la utilización del fruto del mango como recurso alternativo en la alimentación de conejos de engorde.

Palabras clave: Conejos, alimento balanceado comercial, mango, comportamiento productivo.

\section{ABSTRACT}

With the purpose of evaluating the productive behavior of rabbits during the period of fattening growth, I made an experiment that consisted on the partial substitution of the commercial balanced food by the Mango fruit (Mangifera indicates) in a commercial farm of the Monagas state, Venezuela. The experiment lasted 63 days, there were used 32 racially mixed rabbits from New Zealand and California being 30 days of age, lodged in cages. Four treatments were used, with a design completely randomized and four repetitions settled down. T1: 120 commercial balanced food (BFC), T2: 80 BFC g + 40 Mango g, T3: 60 BFC g + 60 Mango g and T4: 40 BFC $g+80$ Mango $g$. The dependent variables were: daily gain of weight average (DGW), final gain of weight $(F G W)$, nutritional conversion with commercial balanced food (NCABFC), final weight at 93 days of age (FP), total consumption (TC). There was made an analysis of variance by the method of square minimums. The results showed significant differences for the variables under study. Being the greater averages in DGW for T1: 26,69 $\pm 1.34 \mathrm{~g}$; FP for T1: 2083,75 $\pm 7.85 \mathrm{~kg}$; TC was for $T 1: 7434,37 \pm 11,23$. The best NCABFC were obtained in the treatments that incorporated the Mango fruit. In general the results obtained allow to infer the use of the fruit as alternative resource in the feeding of fattening rabbits.

Key words: Rabbits, commercial balanced food, Mango, productive behavior.

Ingeniero Producción Animal. Escuela de Zootecnia, Universidad de Oriente, Núcleo Monagas, Venezuela.

2 Profesor Asociado de la Escuela de Zootecnia. Universidad de Oriente. Venezuela. E-mail: ernestohurta@gmail.com. Escuela de Zootecnia, Universidad de Oriente, Núcleo Monagas, Venezuela. 


\section{INTRODUCCIÓN}

La producción de conejos debe ser considerada como una realidad alterna que permitirá satisfacer las necesidades actuales y futuras de alimentación de los sectores más pobres de la población, tanto rural como urbana, principalmente en sistemas caseros de producción donde los conejos pueden aportar cantidades razonables de carne con relativamente poca inversión (Hurtado y Romero, 1999).

La cunicultura representa una alternativa para producir proteína animal de excelente calidad y a bajo costo, sustentada en la alta eficiencia reproductiva del conejo. Debido a que una coneja adulta es capaz de producir 25,2 gazapos destetados anualmente, los cuales al ser llevados al sacrificio se traducen en $48,6 \mathrm{~kg}$ de peso vivo (PV) por coneja por año. Sin embargo, para mantener estos índices en países subdesarrollados debe fortalecerse la investigación en relación a la alimentación de esta especie en estas condiciones (Lukefahr y Cheeke, 1991).

La alimentación de conejos a base de alimentos concentrados comerciales en los países tropicales es altamente costosa y poco conveniente debido a la existencia de un potencial natural, representado por la abundancia de biomasa vegetal, que hasta ahora no ha sido aprovechado de la forma más eficiente. La utilización de cultivos tropicales de alta capacidad de adaptación al medio, en la alimentación de conejos constituye un elemento importante en la construcción de sistemas sostenibles de producción cunícola. Por ello, es necesario entonces identificar recursos alternativos alimenticios con el fin de sustituir parcialmente el alimento concentrado comercial en condiciones tropicales (Nieves y Calderón, 2001).

En el medio tropical $-\mathrm{y}$ especialmente en Venezuela- se cuenta con una gran variedad de fuentes alimenticias con un alto valor biológico que no son utilizadas por otras especies. La alta disponibilidad de plantas probadas o potencialmente útiles para conejos, sustenta la posibilidad de incluirlas en dietas balanceadas preparadas en la granja, para aprovechar la capacidad herbívora de la especie (Nieves et al., 2001).

Diferentes investigaciones han tenido como objetivo sustituir parcialmente al alimento balanceado comercial $(\mathrm{ABC})$ en raciones para conejos por alimentos alternativos (en tal sentido surgen los bloques multinutricionales, inclusión de leguminosas forrajeras y frutas, entre otros) (Nieves y Calderón, 2001).
En países africanos los conejos pueden ser llevados a peso de sacrificio sin el uso de cereales en su ración mediante el uso de leguminosas u otras especies con elevado contenido de proteínas y energía asociadas al uso de subproductos del procesamiento de cereales, como el arroz (Lukefahr y Cheeke, 1990).

El objetivo de la presente investigación es evaluar el comportamiento productivo de conejos durante el período de crecimiento-engorde alimentados con frutos de mango (Mangifera indica) en sustitución parcial del alimento balanceado comercial. Luego, sobre la base de las respuestas obtenidas, se realizará una estimación económica que permita comparar la eficiencia del mango.

\section{MATERIALES Y MÉTODOS}

\section{UBICACIÓN Y CARACTERÍSTICAS DE LA UNIDAD DE PRODUCCIÓN}

El presente estudio se realizó en la finca "TAYU”, ubicada en el sector Chaparral, municipio Piar del estado Monagas, Venezuela. Las coordenadas geográficas son: longitud oeste: $63^{\circ}$ $12^{\prime}$ y latitud norte: $09^{\circ} 07^{\prime}$. El paisaje corresponde a una formación de pie de monte, con temperaturas promedios anuales de $23,7^{\circ} \mathrm{C}$ y precipitaciones de $1.005 \mathrm{~mm}$.

El manejo utilizado en la granja con respecto a la producción cunícola es intensivo, ya que se utiliza alimento balanceado comercial y se llevan los registros de producción de los individuos. Posee un número de animales conformado por 70 madres en producción y 12 reproductores machos y 200 conejos (gazapos y de crecimiento-engorde). Se crían conejos mestizos de las razas Nueva Zelanda y California hasta alcanzar pesos que varían entre 2 y $2,5 \mathrm{~kg}$; la explotación está destinada a la producción de carne de conejo.

\section{MANEJO ALIMENTICIO}

La alimentación se basó en dietas compuestas por alimento balanceado comercial y el fruto del mango criollo fresco, con la composición nutricional que se muestra en la tabla 1 . Se utilizó el fruto de mango con un grado de maduración intermedio a los que se realizó un pequeño corte en la concha antes de ser ofrecidos los animales. 
Tabla 1

Composición nutricional del alimento balanceado comercial (ABC) y el mango criollo

\begin{tabular}{|l|c|c|}
\hline \multicolumn{1}{|c|}{$\begin{array}{c}\text { Valor } \\
\text { nutricional }\end{array}$} & $\begin{array}{c}\text { Alimento balanceado } \\
\text { comercial }(\boldsymbol{\%})\end{array}$ & $\begin{array}{c}\text { Mango criollo } \\
\mathbf{( \% )}\end{array}$ \\
\hline Materia seca & 90,50 & 18,2 \\
\hline Proteína cruda & 14,85 & 2,74 \\
\hline Carbohidratos & 49,73 & 90,10 \\
\hline Grasas & 7,93 & 0,54 \\
\hline Fibra cruda & 16,98 & 3,84 \\
\hline
\end{tabular}

Fuente: Laboratorio de nutrición animal Universidad de Oriente, Monagas, Venezuela.

\section{EXPERIMENTO}

Se utilizaron 32 gazapos de 30 días de edad, con un peso de 402,5 $\pm 28,61$ durante 63 días en jaulas de crianza de $0,80 \times 0,60 \times 0,40 \mathrm{~m}$, entre los meses de junio, julio y agosto del año 2005 , en un diseño completamente aleatorizado con cuatro tratamientos y cuatro repeticiones, teniendo como covariable al peso inicial, con dos conejos por unidad experimental. Los tratamientos fueron los siguientes: $\mathrm{T} 1 \mathrm{l} 120 \mathrm{~g}$ alimento balanceado comercial (ABC); T2: $80 \mathrm{~g} \mathrm{ABC}+40 \mathrm{~g}$ mango; $\mathrm{T} 3$ : $60 \mathrm{~g} \mathrm{ABC}+60 \mathrm{~g}$ mango y $\mathrm{T} 4: 40 \mathrm{~g} \mathrm{ABC}+80 \mathrm{~g}$ mango.

Las variables bajo estudio medidas fueron:

- Ganancia diaria de peso promedio (GDP): Se estableció por la diferencia entre el peso final y el peso inicial de la semana dividido entre siete días, durante todo el ensayo.

- Consumo total durante el ensayo (CONSTOTAL): Se refiere al consumo de alimento total al final del ensayo por animal. El cual es la suma de todos los consumos diarios hasta la edad de sacrificio y se obtuvieron por diferencia de pesos entre el alimento ofrecido y el rechazado.

- Peso final (PESOFIN): El peso alcanzado a los 93 días de edad.

- Conversión alimenticia con alimento balanceado comercial (CAABC): Se calculó a través de la fórmula (De Blas 1989):

- $\mathrm{CAABC}=($ consumo de alimento $(\mathrm{kg})) /($ peso final - peso inicial (kg)) expresada en $\mathrm{kg}$ de alimento consumido $\mathrm{x} \mathrm{kg}$ de peso vivo obtenido del destete hasta los 93 días de edad.

\section{ANÁLISIS ESTADÍSTICO}

Los datos se analizaron mediante un análisis de varianza (SAS, 1998) con un modelo de clasificación simple que tuvo a los tratamientos como única causa de variación y como covariable el peso inicial. Se realizó la prueba de medias a través del método de Duncan al 5\%, para las variables que resultaron significativas.

\section{RESULTADOS Y DISCUSIÓN}

El análisis de varianza para la ganancia diaria de peso, peso final y consumo total arrojó diferencias estadísticamente significativas entre tratamientos, al igual el efecto significativo que tuvo el peso inicial como covariable. En la tabla 2 se presentan las medias con respecto a los tratamientos, observándose que la mejor respuesta la obtuvieron los animales alimentados con el tratamiento 1 (120 g ABC). Sin embargo, se encontraron valores aceptables para el tratamiento 2 ( $80 \mathrm{~g} \mathrm{ABC}+40 \mathrm{~g}$ Mango).

Con respecto a la ganancia diaria promedio, estos resultados superan a los niveles reportados por cu Cheeke y Raharjo (1988) cuando utilizaron ramio blanco (Boehmeria nivea, $L$ Gaud) en un $25 \%$ y $75 \%$ de $\mathrm{ABC}$ como alternativa para disminuir los costos de alimentación en los sistemas de producción de conejos (19,241 g/animal/día), y a los reportados por Nieves y Calderón (2001) en dietas no convencionales con Trichanthera gigantea cuando se suministró ad libitum y lombriz roja californiana (Eisenia foetida) (en un 2\% de inclusión) 19,29 g/animal/día. Entretanto, para el peso final los valores obtenidos para el tratamiento $2(1.861,50 \mathrm{~g})$ son aceptables debido a la inclusión del mango criollo y a la reducción de costos que la sustitución representa en la alimentación de conejos.

Los consumos totales estuvieron dentro de lo normal. Se observó un consumo total de alimento balanceado comercial en los tratamientos en donde se incluía el fruto de mango, además del consumo de la fuente energética. Es importante señalar que no se presentaron problemas digestivos en los animales, teniéndose registrado cero mortalidad.

Los valores obtenidos corroboran lo reportado en la literatura sobre la adaptabilidad de esta especie a distintos recursos alternativos que pueden ser viables en la alimentación de conejos de engorde. 
Los tratamientos arrojaron un efecto significativo $(\mathrm{p}<0,05)$ para la variable conversión alimenticia con el alimento balanceado comercial. Se observan en la tabla 3 los promedios obtenidos, siendo los mejores los que incorporaron en la ración el fruto de mango. Esto refleja el efecto nutricional que este recurso aportó a los conejos en esta fase de producción bajo estas condiciones.

\section{Tabla 3}

Promedios y errores estándar de la conversión alimenticia con alimento balanceado comercial

\begin{tabular}{|c|c|}
\hline Tratamiento & $\begin{array}{c}\text { Conversión Alimento Balanceado } \\
\text { Comercial }(\mathbf{k g} / \mathbf{k g})\end{array}$ \\
\hline $\mathrm{T} 1$ & $4,43 \pm 0,07^{\mathrm{a}}$ \\
\hline $\mathrm{T} 2$ & $3,24 \pm 0,08^{\mathrm{b}}$ \\
\hline $\mathrm{T} 3$ & $2,41 \pm 0,02^{\mathrm{c}}$ \\
\hline $\mathrm{T} 4$ & $2,13 \pm 0,06^{\mathrm{d}}$ \\
\hline
\end{tabular}

abcd: medias con letras diferentes entre columnas difieren estadísticamente a una probabilidad menor a $5 \%$.
Estos valores son superiores a los señalados por Morales et al. (2002) $(3,30 \mathrm{~kg} / \mathrm{kg})$ cuando utilizaron Leucaena leucocephala con niveles de $25 \%$ de inclusión en dietas para conejos de engorde. Sin embargo, los resultados obtenidos proporcionan una aceptable conversión alimenticia debido a la alta aceptación del mango por los conejos. Es necesario destacar que a medida que aumentan los niveles de inclusión del mango criollo, la conversión con alimento balanceado comercial (CAABC) disminuye considerablemente, lo que refleja una reducción importante en los gastos de alimentación de la producción cunícola.

\section{CONCLUSIONES}

La mejor respuesta productiva la obtuvieron los animales que consumieron la dieta correspondiente al tratamiento 1 (120 g ABC). Sin embargo, el comportamiento de los animales al tratamiento $2(80 \mathrm{~g}$ $\mathrm{ABC}+40 \mathrm{~g}$ mango) se considera admisible para estas condiciones. Además existe alta aceptación del fruto del mango criollo por parte de los conejos, lo que indica la factibilidad de utilizarlo en la etapa de engorde.

Tabla 2

Promedios y errores estándar de las variables ganancia diaria de peso, peso final y consumo total

\begin{tabular}{|c|c|c|c|}
\hline Tratamiento & GDP $(\mathbf{g})$ & Peso Final $(\mathbf{g})$ & Consumo Total $(\mathbf{g})$ \\
\hline T1 & $26,69 \pm 1,34^{\mathrm{a}}$ & $2083,75 \pm 7,85$ & $7434,37 \pm 6,80^{\mathrm{a}}$ \\
\hline T2 & $23,46 \pm 1,56^{\mathrm{b}}$ & $1861,50 \pm 11,06^{\mathrm{b}}$ & $7251,88 \pm 28,71^{\mathrm{b}}$ \\
\hline T3 & $22,00 \pm 1,40^{\mathrm{b}}$ & $1736,00 \pm 6,47^{\mathrm{b}}$ & $6677,5 \pm 44,78^{\mathrm{b}}$ \\
\hline T4 & $19,98 \pm 1,17^{\mathrm{c}}$ & $1652,50 \pm 18,43^{\mathrm{c}}$ & $7087,88 \pm 2,55^{\mathrm{c}}$ \\
\hline
\end{tabular}

abc: medias con letras diferentes entre columnas difieren estadísticamente a una probabilidad menor a $5 \%$.

\section{LITERATURA CITADA}

CHEEKE, P.; RAHARJO, Y. 1988. Evaluación de forrajes tropicales y subproductos agrícolas como alimento para conejos. Memorias CIPAV (1): 33-41.

DE BLAS, J. 1989. Alimentación del Conejo. 2da edición. Mundi prensa (5): 43-49, 139.

HURTADO, E.; ROMERO, R. 1999. Efectos no genéticos sobre el comportamiento productivo de conejos (Oryctolagus cuniculus) durante el crecimiento post destete. Revista Fac. Ciencias Veterinarias. UCV, Maracay, Venezuela (1): $139-142$
LUKEFAHR, S.; CHEEKE, P. 1990. Rabbit project planning strategies for developing countries, 1: practical considerations. Livestock. Research for Rural Development (1): 2-9.

LUKEFAHR, S.; CHEEKE, P. 1991. Rabbit project development strategies insubsistence farming system. Editor s. s. Branckaert. World Animal Review a Quarterly Journal on Animal Health, Production and Products FAO (2): 69.

MORALES, M.; JUÁREZ, A.; ÁVILA M.; FUENTES, B.; VELÁSQUEZ, G. 2002. Efectos de la suplementación 
con forraje verde hidropónico de cebada en los indicadores productivos y el rendimiento en canal de conejos Nueva Zelanda durante la fase de engorde. II congreso de cunicultura de las Américas. pp. 03-85

NIEVES, D.; CALDERÓN, J. 2001. Inclusión de harina de lombriz (Eisenia foetida) en dietas no convencionales y suplementación con Trichanthera gigantea en conejos de engorde. Investigación agrícola. [Documento en Línea] disponible: http://www.redpavfpolar.info.ve/danac/volumen6/art4/index.html. [Consulta: 18-03-2007].

NIEVES, D.; LÓPEZ, D.; CADENA, D. 2001. Alimentación de conejos de engorde con dietas basadas en materias primas no convencionales y suplementación con Trichanthera gigantea. Revista UNELLEZ, Guanare. (2): 01-14.

SAS. 1998. SAS User's guide: Statistics. SAS Inst., Inc., Car., N.C. 